\title{
Gene-environment interaction with smoking for increased non- muscle-invasive bladder cancer tumor size
}

\author{
Nadezda Lipunova ${ }^{1,2,3}$, Anke Wesselius ${ }^{3}$, Kar K. Cheng ${ }^{4}$, Frederik-Jan van Schooten ${ }^{5}$, Richard T. Bryan ${ }^{1}$, \\ Jean-Baptiste Cazier ${ }^{1,2}$, Maurice P. Zeegers ${ }^{1,3}$ \\ ${ }^{1}$ Institute of Cancer and Genomic Sciences, ${ }^{2}$ Centre for Computational Biology, University of Birmingham, Birmingham, UK; ${ }^{3}$ Department \\ of Complex Genetics, Maastricht University, Mastricht, The Netherlands; ${ }^{4}$ Institute for Applied Health Research, University of Birmingham, \\ Birmingham, UK; ${ }^{5}$ Department of Genetic and Molecular Toxicology, Maastricht University, Maastricht, The Netherlands \\ Contributions: (I) Conception and design: N Lipunova, RT Bryan, MP Zeegers, A Wesselius; (II) Administrative support: N Lipunova, RT Bryan, \\ A Wesselius; (III) Provision of study materials or patients: N Lipunova, RT Bryan, MP Zeegers, KK Cheng; (IV) Collection and assembly of data: \\ N Lipunova, RT Bryan, MP Zeegers, KK Cheng; (V) Data analysis and interpretation: N Lipunova, RT Bryan, A Wesselius, JB Cazier, FJ van \\ Schooten; (VI) Manuscript writing: All authors; (VII) Final approval of manuscript: All authors. \\ Correspondence to: Nadezda Lipunova. Institute of Cancer and Genomic Sciences, Centre for Computational Biology, University of Birmingham, \\ Birmingham, UK; Department of Complex Genetics, Maastricht University, Maastricht, The Netherlands. Email: nxl689@student.bham.ac.uk; \\ n.lipunova@maastrichtuniversity.nl.
}

Background: Urinary bladder cancer (UBC) is one of few cancers with an established gene-environment interaction $(\mathrm{GxE})$ with smoking. However, it is unknown whether the interaction with tobacco use is present non-muscle invasive bladder cancer (NMIBC) and characteristics of prognostic relevance. We aimed to investigate if smoking status and/or smoking intensity interact with the effect of discovered variants on key NMIBC characteristics of tumor grade, stage, size, and patient age within the Bladder Cancer Prognosis Programme (BCPP) cohort.

Methods: Analyzed sample consisted of 546 NMIBC patients with valid smoking data from the BCPP. In a previous genome-wide association study (GWAS), we have identified 61 single nucleotide polymorphisms (SNPs) potentially associated with the NMIBC characteristics of tumor stage, grade, size, and patient age. In the current analysis, we have tested these SNPs for GxE with smoking.

Results: Out of 61 SNPs, 10 have showed suggestion (statistical significance level of $\mathrm{P}<0.05$ ) for $\mathrm{GxE}$ with NMIBC tumor size rs35225990, rs188958632, rs180910528, rs74603364, rs187040828, rs144383242, rs117587674, rs113705641, rs2937268, and chromosome 14:38247577. All SNPs were located across loci of $1 \mathrm{p} 31.3,3 \mathrm{p} 26.1,6 \mathrm{q} 14.1,14 \mathrm{q} 21.1$, and 13q14.13. In addition, two of the tested polymorphisms were suggestive for interaction with smoking intensity (chromosome 14:38247577 and rs2937268).

Conclusions: Our study suggests interaction between genetic variance and smoking behavior for increased NMIBC tumor size at the time of diagnosis. Further replication is required to validate these findings.

Keywords: Bladder cancer; gene-environment interaction (GxE); smoking; SNP; tumor size

Submitted Sep 19, 2019. Accepted for publication Feb 14, 2020.

doi: $10.21037 /$ tau-19-523

View this article at: http://dx.doi.org/10.21037/tau-19-523

\section{Introduction}

Current evidence suggests that the effect of urinary bladder cancer (UBC) related single nucleotide polymorphisms (SNPs) may depend on smoking exposure (1-4). However, it remains unknown if gene-environment interactions $(\mathrm{GxE})$ can be differential across UBC subtypes and characteristics.

To date, studies on gene-tobacco interactions (GxT) for UBC have only considered the overall risk of UBC as an outcome. These investigations have resulted in overlapping findings, collectively reporting multiple significant loci 
(2-4). Some of the most commonly reported genes (NAT2, UGT1A6) are regulatory of phase II detoxification, a pathway for metabolizing tobacco-related carcinogens (i.e., aromatic amines) $(2,3,5)$. As polymorphisms in NAT2 and UGT1A6 alter smoking-related carcinogen clearance, their effect naturally becomes limited to smokers. Concurrently, the effect is reduced (2) or almost absent for never smokers (5). Importantly, the level of smoking exposure has shown to be an important aspect in GxT, revealing an important pattern of certain genotypes interacting with smoking in a dose-dependent manner $(2,4-7)$. Some genotypes, namely GSTM1-null status (3), appear more important for non-smokers, although significant effects are present regardless of tobacco use. Expectedly, the effect of these genotypes on UBC risk does not vary within smoking intensity categories (6).

UBC is one of few cancers with an established GxE (4), yet previous research has not distinguished between muscle invasive bladder cancer (MIBC) and non-muscle invasive bladder cancer (NMIBC) groupings. Given our continuallyevolving knowledge of the pathogenesis of MIBC and NMIBC (8), not stratifying for these categories in genetic analyses could result in overlooking important biological mechanisms. Furthermore, it is now known that genetic loci for UBC risk are not relevant for UBC prognosis (9), which continues to highlight the complexity of genetic associations. Finally, little evidence is present for genetic associations with specific characteristics such as tumor size, grade, stage or patient age $(2,5)$. However, these characteristics are especially influential for NMIBC outcomes (10), and are more specific entities than the more broadly-defined prognostic outcomes such as recurrence or progression.

We have previously carried out a genome-wide association study (GWAS) on NMIBC tumor and patient characteristics in the Bladder Cancer Prognosis Programme (BCPP) cohort, potentially identifying novel genetic associations (11). In the current study, we have investigated if smoking status and/ or smoking intensity interact with the effect of discovered variants on key NMIBC characteristics of tumor grade, stage, size and patient age within the BCPP cohort.

\section{Methods}

\section{Participants and genotyping}

BCPP is a prospective cohort of 1,544 participants (12). Baseline clinical tumor characteristics (stage, grade, size) were collected from medical records, while demographic and smoking data were retrieved from records of semistructured interviews conducted at baseline. Largest tumor size was estimated visually during cystoscopy. Blood samples of 888 participants were genotyped on the Illumina Infinium OmniExpress-24 BeadChip array (previously known as The HumanOmniExxpress-24 BeadChip) at deCODE genetics (Reykjavik, Iceland) (13).

\section{Quality control (QC)}

QC for the initial GWAS was carried out in PLINK v1.90 (released $17^{\text {th }}$ November 2016) $(14,15)$ and is described in more detail elsewhere (11). In brief, genotype samples yielding inconclusive sex calls, excessive missingness, low or increased heterozygosity rate were excluded from the analysis. Related individuals and participants presenting as population outliers [identified in the principal component analysis (PCA)] were additionally excluded from the study. Genetic markers deviating from the Hardy-Weinberg equilibrium (HWE), those with high missingness rate and low minor allele frequency (MAF) were excluded. All QC steps resulted in a pre-imputed dataset consisting of 653 individuals and 597,764 SNPs.

\section{Imputation}

A two-step imputation was performed with Eagle v2.3.2 (16) for haplotype phasing and IMPUTE2 (17) for genotype imputation, using 1000 Genomes Phase 3 (released $2^{\text {nd }}$ May 2013) (18) as a reference panel in the genome build 19 (GRCh37/hg19). Post-imputation QC consisted of deleting markers having info score of $<0.3$ and MAFs of $<1 \%$, resulting in a dataset containing $11,914,228$ markers that were used for GWAS analysis with tumor and patient baseline characteristics. The exact thresholds applied and number of exclusions per step are outlined in detail in Figure 1.

\section{Outcomes and exposure variables}

As studies on genetic associations with NMIBC baseline characteristics are scarce, we have used results of a previously-reported GWAS in the BCPP cohort (11). Hence, a total of 61 discovered variants were used to test for GxT. The outcomes were as follows: tumor size (centimeters), stage (T1 vs. Ta/Tis), grade (G3/G2 vs. G1), and patients' age [years as a continuous outcome and a binary variable with sample mean as a cut-off value $(</ \geq 69.9$ years, representing sample mean age)]. Our analyses were restricted to NMIBC 


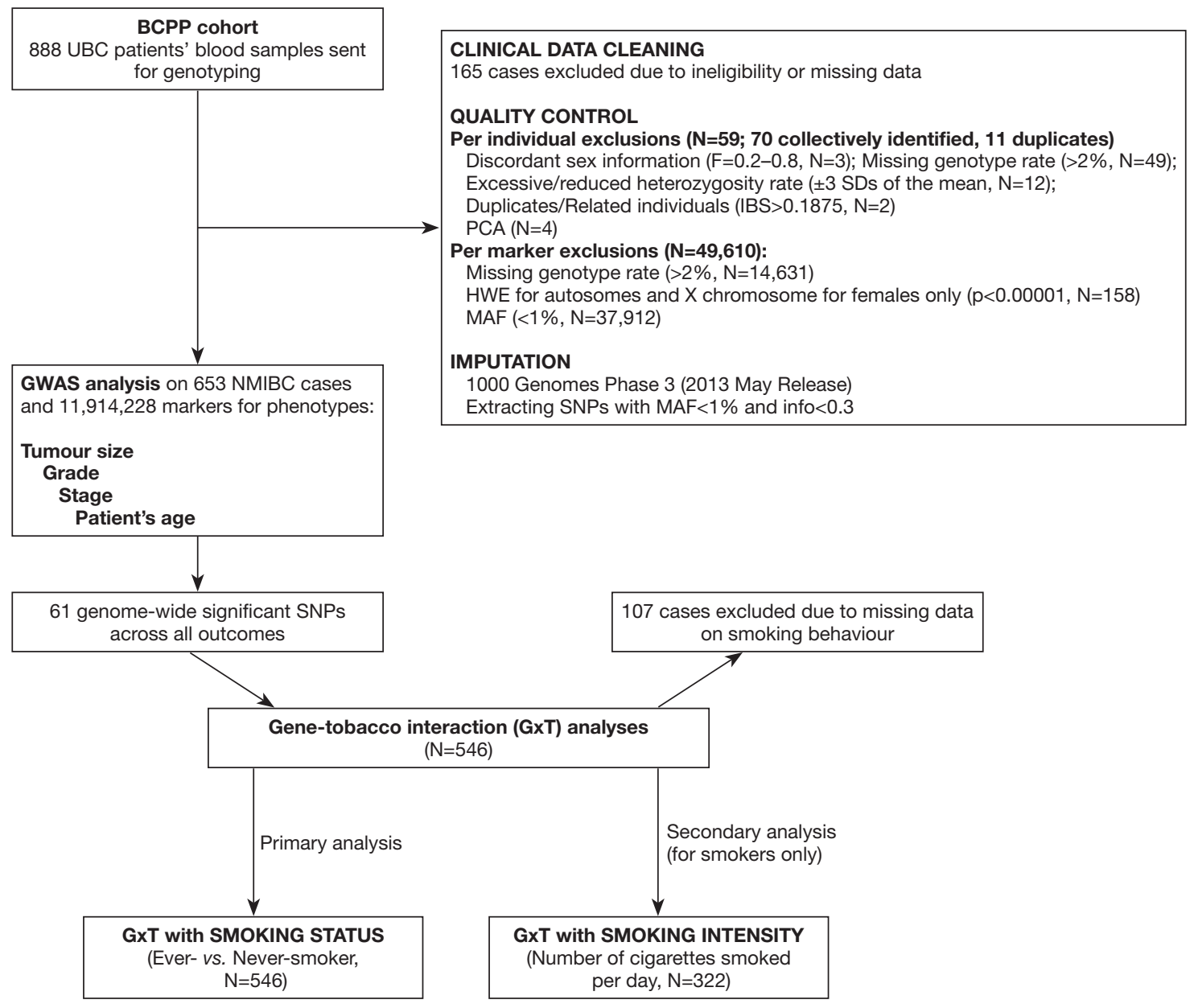

Figure 1 Flowchart for data analysis on GxT in the BCPP cohort. GxT, gene-tobacco interaction; BCPP, Bladder Cancer Prognosis Programme; HWE, Hardy-Weinberg equilibrium; SD, standard deviation; IBS, identity by state; PCA, principal component analysis; MAF, minor allele frequency; NMIBC, non-muscle invasive bladder cancer; PCA, principal component analysis; SD, standard deviation; SNP, single nucleotide polymorphism.

cases only (corresponding to recorded stage at the time of diagnosis of Ta, T1, or Tis) who also had valid records on tobacco use, resulting in 546 patients.

Smoking exposure was modelled as two variables: smoking status (ever $v s$. never smokers) and smoking intensity (number of cigarettes smoked daily for smokers only). GxT with smoking status was considered as a primary analysis (including 546 subjects), while analysis on smoking intensity was considered secondary (consisting of 322 smokers with valid data on number of cigarettes smoked per day).

\section{Statistical analysis}

Differences in baseline sample characteristics were calculated by applying chi-square distribution test for categorical variables (age, tumor size, stage, grade) and $t$-test for continuous variables (age, tumor size, smoking intensity).

To test the interaction terms that account for the uncertainty of imputed genotypes, QUICKTEST software was used (19). All analyses were adjusted for gender and first five genetic principal components to increase estimate precision.

A total set of 61 SNPs that were discovered in the previous GWAS analysis of the BCPP cohort (11), were tested. GxT term (depicted as SNP $\times$ smoking or SNP $\times$ smoking intensity) was modelled for each discovered association. An interaction term was deemed to be significant if $\mathrm{P}$ value $<0.05$. 
Table 1 Descriptive characteristics of the BCPP sample for GxE with smoking analysis

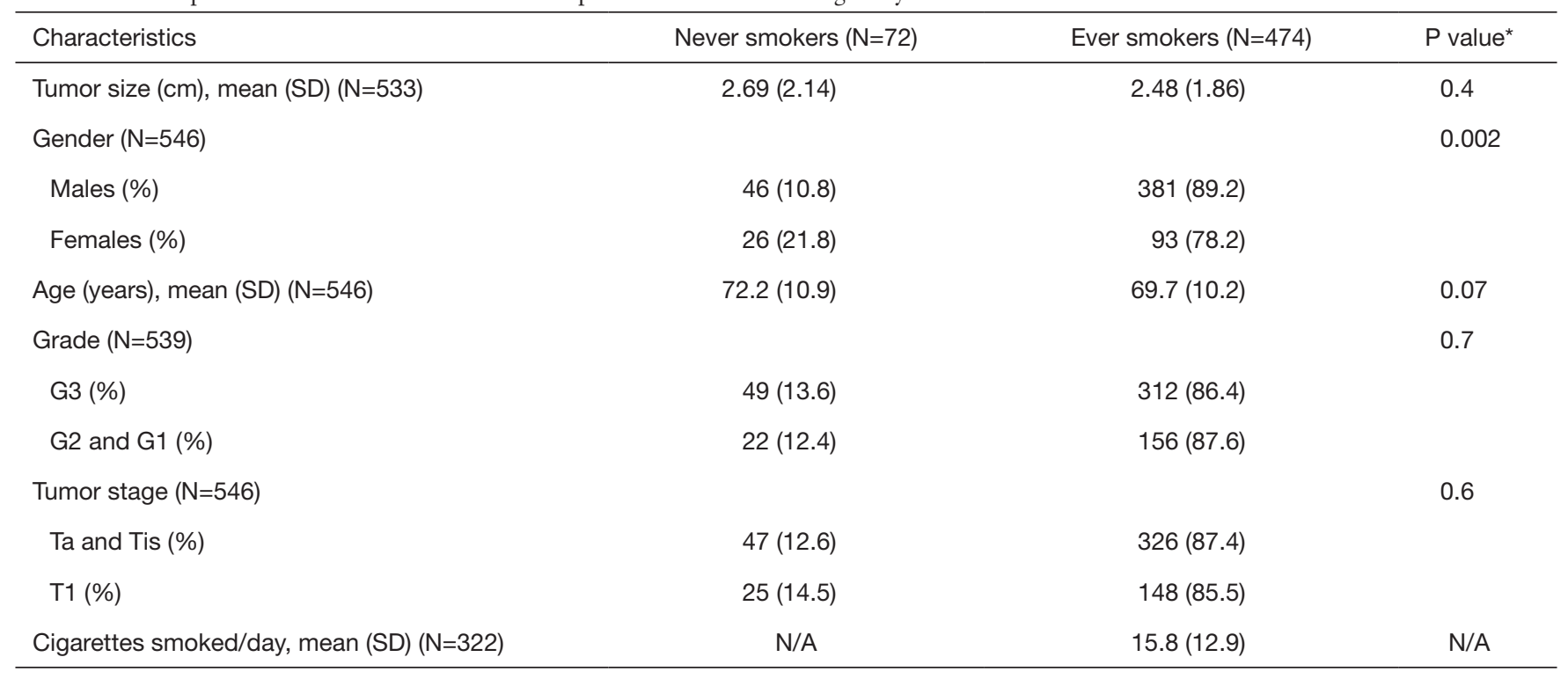

*, $\mathrm{P}$ value was calculated with chi-square test for categorical variables and t-test for continuous outcomes. BCPP, Bladder Cancer Prognosis Programme; GxE, gene-environment interaction.

\section{Functional annotation and network analysis}

All identified significant SNPs were annotated to an overlapping or closest gene using a web-based SNPnexus tool $(20,21)$, with Ensembl (22) (version 74) as a functional annotation system. Gene-Tissue Expression (GTEx) database (23) was queried for all significant SNPs to identify expression quantitative loci (eQTL).

\section{Results}

BCPP patient characteristics are presented in Table 1. Out of the total sample of 546 participants, 72 were neversmokers, whilst 474 had a history of tobacco use. Participant gender was predominantly male (chi-square statistic $=9.97$, $\mathrm{P}=0.002)$. No other statistically significant differences in baseline characteristics between smokers and never-smokers were observed.

Out of 61 SNPs tested for a GxT in the BCPP cohort, 10 have reached $\mathrm{P}<0.05$, all associated with tumor size (Table 2, Figure 2). Five of these SNPs were located in 6q14.1, two in $14 \mathrm{q} 21.1$; while the rest were mapped to $1 \mathrm{p} 31.3,3 \mathrm{p} 26.1$, and $13 \mathrm{q} 14.13$.

The most significant interaction with smoking status was observed for a SNP in $14 \mathrm{q} 21.1$ locus [base pair (BP): $14: 38247577, \mathrm{P}=0.0008]$, that maps to the TTC6 gene (Table 3). Another SNP in the same locus has also yielded statistical significance for interaction (rs188958632, $\mathrm{P}=0.008$, TTC6), but the two variants show differential results for smokers and non-smokers (Figure 2). SNP in 14:38247577 has an effect on tumor size only among smokers $(\beta=7.1 \mathrm{~cm}, \mathrm{P}=1.93 \mathrm{E}-13)$. However, rs 188958632 is significant among both tobacco use groups, with a larger effect size for never-smokers $[\beta$ (never-smokers) $=9.9 \mathrm{~cm}$, $\mathrm{P}=9.84 \mathrm{E}-05 ; \beta$ (ever-smokers $)=2.6 \mathrm{~cm}, \mathrm{P}=6.98 \mathrm{E}-07]$ (Table 2, Figure 2).

Five variants on $6 \mathrm{q} 14.1$ were statistically significantly associated with NMIBC tumor size among both strata of smoking behavior; however, $\beta$ estimates for never-smokers were universally higher, more than doubling the effect sizes for ever-smokers (Table 2, Figure 2). All five SNPs (rs180910528, rs74603364, rs187040828, rs144383242, and rs117587674) have mapped to intergenic regions (Table 3). Interestingly, two of these SNPs (rs144383242 and rs117587674) are recorded in the GTEx database as having an effect on the expression of HMGN3-AS1 in the tibial nerve tissue (Figures $S 1, S 2$, available online) (23). None of the 6q14.1-located SNPs have shown significant interaction between tumor size and smoking intensity in our sample.

Rs113705641 on 3p26.1 (intergenic region) has shown to only be statistically significantly associated with tumor size among ever-smokers $(\beta=2.4 \mathrm{~cm}, \mathrm{P}=2.39 \mathrm{E}-06)$, and no interaction with smoking intensity. Similarly, rs2937268 on 1p31.3, mapped to the PDE4B gene, has not only reached 


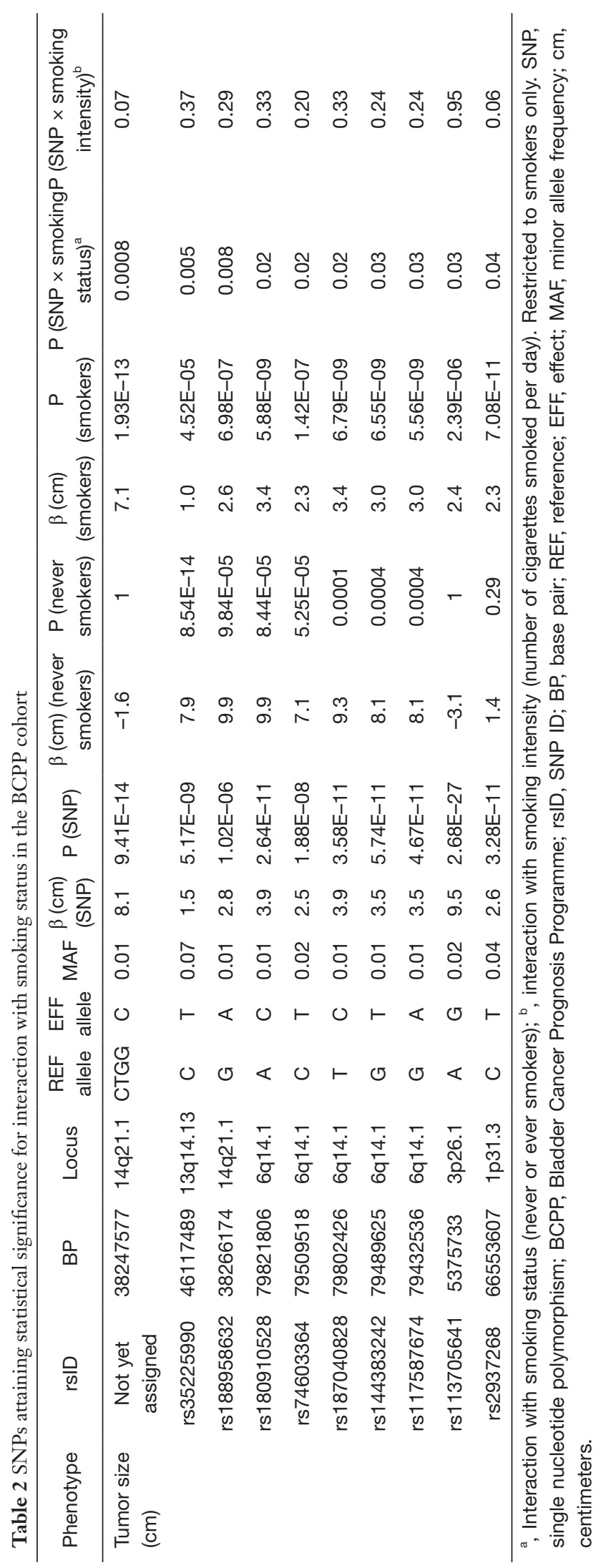

significance among ever-smokers $(\beta=2.3 \mathrm{~cm}, \mathrm{P}=7.08 \mathrm{E}-11)$, but has also shown suggestive interaction with smoking intensity $(\mathrm{P}=0.06)$ among tobacco users.

It is notable that variants associated with a stronger effect among never-smokers, had higher estimates overall (ranging from 7.1 to $9.9 \mathrm{~cm})$. In contrast, rs2937268 (1p31.3, PDE4B) and rs113705641 (3p26.1) variants, shown to be significant only for smokers, have been observed to carry a more modest increase in tumor size. The exception would be the $14: 38247577$ variant in $14 \mathrm{q} 21.1$ that was not significant among never-smokers, but resulted in a very large tumor size increase of $7.1 \mathrm{~cm}$ in smokers (Table 2, Figure 2).

\section{Discussion}

We hereby report findings for $\mathrm{GxE}$ with smoking status for SNPs previously discovered in a GWAS on NMIBC baseline characteristics (11). The study provides indication for ten variants interacting with smoking status for tumor size at the time of NMIBC diagnosis, and two of those additionally having suggestive interactions with smoking intensity.

Previous studies on GxT for UBC risk show repeating patterns that highlight potential biological mechanisms $(2,4,24)$. Smoker-specific UBC risk genes are associated with pathways of metabolite detoxification (24), offering a plausible explanation on why the effect is more penetrant among smokers. Alternatively, genes having more importance among never smokers are more often enriched in those regulating cell cycle and DNA integrity (24). Additionally, results across multiple GxT studies on UBC demonstrate that variants significant for never-smokers carry a large effect (i.e., multiplicative interaction), while SNPs that are more important for smokers usually exhibit a milder effect (additive interaction) (5).

Among smokers, the strongest evidence for GxT in our sample was observed for variants rs113705641 (3p26.1) and rs2937268 (1p31.3, PDE4B).

Rs113705641 maps to a long non-coding RNA molecule, and reports on its function are lacking, therefore we are unable to postulate on a potential biological mechanism of interaction.

On the other hand, phosphodiesterase 4B (PDE4B) enzymes are well described to regulate cyclic adenosine 3', 5'-monophosphate (cAMP) concentration in cells by breakdown to non-active molecules (25). The PDE4 family is cAMP-specific, and four existing isoforms of PDE4B account for most cAMP-degradation in a cell (25). 


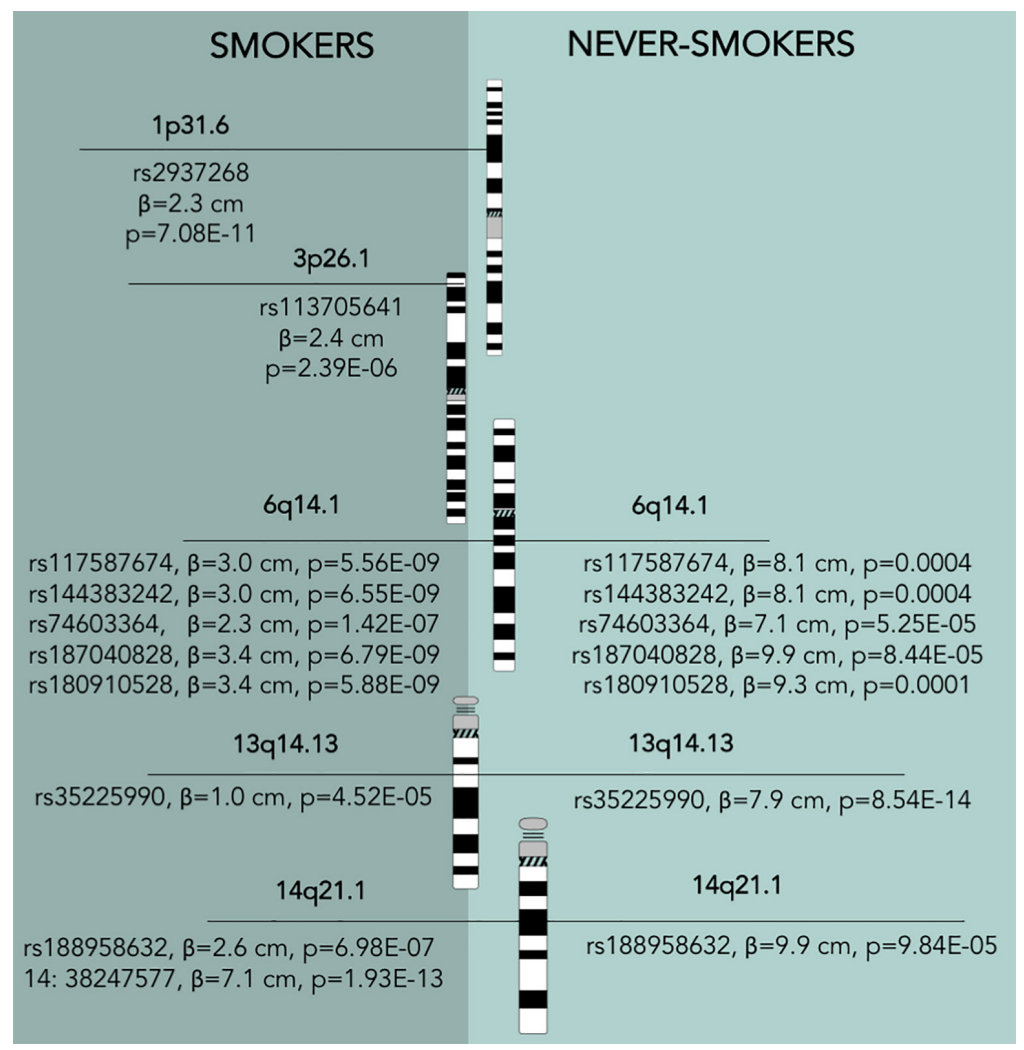

Figure 2 Group-specific patterns of significant loci for gene-environment interaction for NMIBC tumour size in the BCPP cohort. Idiogram credit: David Adler, Available online: http://www.pathology.washington.edu/research/cytopages/idiograms/human/. NMIBC, nonmuscle invasive bladder cancer; BCPP, Bladder Cancer Prognosis Programme.

The beneficial effect of PDE inhibitors is well-known and they are commonly used for a variety of disorders: inflammatory conditions [namely chronic obstructive pulmonary disease (COPD) (26,27), asthma (26), and psoriasis $(26,27)]$, overactive bladder $(28,29)$, and various cancer types (30-35). Importantly, studies suggest tumor PDE4 expression can serve as a prognostic marker in colorectal cancer (35). As cAMP signaling is pleiotropic, the mechanisms behind the observed benefit have garnered more than one explanation. For example, elevated cAMP levels suppress cell invasion and migration by disrupting the microtubule cytoskeleton in bladder cancer cells (36), increase phosphatidylinositol 3-kinase (PI3K/AKT)-dependent apoptosis in B-cell malignancies (37), and prohibit proliferation via protein kinase (PKA) and cAMP response element binding (CREB) protein pathways in ovarian cancer (38).

Interestingly, tobacco exposure also increases cAMP signaling in epithelial cells (39), specifically by polycyclic aromatic hydrocarbons (PAHs, found in tobacco smoke) binding to aryl hydrocarbon receptors (AhR) (39). Stimulation of cAMP results in increased levels of amphiregulin (AR), which is hypothesized to eventually form a self-sustainable loop of survival for neoplastic cells (39-41).

In our analysis, cAMP seems to be the overlapping component between $P D E 4 B$ and smoking, which results in a counter-effect of cAMP regulation (i.e., overexpressed PDE4B decreases cAMP, while smoking increases cAMP). Thus, we hypothesize that smoking interacts with $P D E 4 B$ mutation status and results in a smaller tumor size increase than the PDE $4 B$ mutation itself. This pattern would explain the difference between a main effect exhibited by the SNP in $P D E 4 B$ (resulting in $2.6 \mathrm{~cm}$ increase in tumor size) and a surprising reduced estimate of a joint effect of the SNP and smoking exposure ( $2.1 \mathrm{~cm}$ increase in tumor size).

Smoking, when considered as an independent factor, is the main external risk factor for developing UBC (42). Our described interaction is conditional on the overexpression of PDE4B enzyme; thus, it should be viewed not as a case for the benefits of smoking, but one describing a potential cAMP-related pathway of bladder cancer pathogenesis. 




An additional variant in 14q21.1 (BP: 38247577) reached statistical significance among smokers only. However, a SNP in the same locus (rs188958632) showed contradicting results and had a significant effect regardless of smoking exposure. We suspect that the SNP on 14:38247577 is more likely to be a random result due to a lower imputation accuracy (info $=0.59$ ) than $\mathrm{rs} 188958632($ info $=0.95)$ (remaining SNPs have an info score of $\geq 0.90$, with the exception for rs113705641, with info $=0.62$ ).

In contrast, variants on $13 \mathrm{q} 14.13$ and $6 \mathrm{q} 14.1$ have reached significant values for main effects among all participants. However, the clinical importance might be higher for never smokers as they showed substantially higher effect sizes than for smokers. The mapped FAM194B (ERICH6B) gene $(13 \mathrm{q} 14.13)$ is recorded in the GTEx database as excessively expressed in testis tissue (23), but other reports on gene function or phenotype associations are scarce, thus precluding a discussion on its potential biological pathway. SNPs in 6q14.1 were mapped to intergenic regions, with rs180910528 and rs187040828 located between protein coding genes of PHIP and $H M G N 3$. Interestingly, four of the total five SNPs in 6q14 region (rs180910528, rs187040828, rs144383242, and rs117587674) are recorded in the GTEx database as having a cis-regulatory effect on an RNA gene of HMGN3-AS1 (23). Antisense RNAs bind to messenger RNA molecules, preventing them from being translated into protein (43). Thus, lower expression of $H M G N 3-A S 1$ may cause higher levels of proteins coded by HMGN3 gene. HMGN is a family of nucleosome-binding proteins that alter chromatin structure and regulate essential cell functions, such as differentiation and development (44).

Our study has several limitations. Current analysis was carried out on a relatively small sample of individuals, and false-positives are naturally not to be excluded. The associations between genetic variation and NMIBC tumor size should be interpreted with caution, as they lack validation (11). Besides tobacco, other variables, such as occupational-related exposures with aromatic amines might have independent $\mathrm{GxE}$ effects and were not adjusted for in our analyses (45). Larger samples with detailed clinical data are a next step to exploring and validating reported GxEs with smoking for NMIBC. Until robust evidence exists for including genetic variance into NMIBC prognostication tools, our study is complementary to other proof-of-principle reports (2).

In summary, our study suggests smoking might alter the outcome between genetic variance and NMIBC tumor characteristics. These results may provide more evidence on 
the complexity of the joint influence of genetic background and external exposures on bladder cancer.

\section{Acknowledgments}

Funding: None.

\section{Footnote}

Conflicts of Interest: All authors have completed the ICMJE uniform disclosure form (available at http://dx.doi. org/10.21037/tau-19-523). RTB has contributed to advisory boards for Olympus Medical Systems and Janssen. The other authors have no conflicts of interest to declare.

Ethical Statement: The authors are accountable for all aspects of the work in ensuring that questions related to the accuracy or integrity of any part of the work are appropriately investigated and resolved. All procedures performed in studies involving human participants were in accordance with the ethical standards of the institutional and/ or national research committee and with the 1964 Helsinki declaration and its later amendments or comparable ethical standards. Informed consent was obtained from all individual participants included in the study.

Open Access Statement: This is an Open Access article distributed in accordance with the Creative Commons Attribution-NonCommercial-NoDerivs 4.0 International License (CC BY-NC-ND 4.0), which permits the noncommercial replication and distribution of the article with the strict proviso that no changes or edits are made and the original work is properly cited (including links to both the formal publication through the relevant DOI and the license). See: https://creativecommons.org/licenses/by-nc-nd/4.0/.

\section{References}

1. Selinski S. The post GWAS era: strategies to identify gene-gene and gene-environment interactions in urinary bladder cancer. EXCLI J 2014;13:1198-203.

2. Garcia-Closas M, Rothman N, Figueroa JD, et al. Common genetic polymorphisms modify the effect of smoking on absolute risk of bladder cancer. Cancer Res 2013;73:2211-20.

3. Rothman N, Garcia-Closas M, Chatterjee N, et al. A multi-stage genome-wide association study of bladder cancer identifies multiple susceptibility loci. Nat Genet 2010;42:978-84.
4. Figueroa JD, Han SS, Garcia-Closas M, et al. Genomewide interaction study of smoking and bladder cancer risk. Carcinogenesis 2014;35:1737-44.

5. García-Closas M, Malats N, Silverman D, et al. NAT2 slow acetylation, GSTM1 null genotype, and risk of bladder cancer: results from the Spanish Bladder Cancer Study and meta-analyses. Lancet 2005;366:649-59.

6. Moore LE, Baris DR, Figueroa JD, et al. GSTM1 null and NAT2 slow acetylation genotypes, smoking intensity and bladder cancer risk: results from the New England bladder cancer study and NAT2 meta-analysis. Carcinogenesis 2011;32:182-9.

7. Lubin JH, Kogevinas M, Silverman D, et al. Evidence for an intensity-dependent interaction of NAT2 acetylation genotype and cigarette smoking in the Spanish Bladder Cancer Study. Int J Epidemiol 2007;36:236-41.

8. Czerniak B, Dinney C, McConkey D. Origins of bladder cancer. Annu Rev Pathol 2016;11:149-74.

9. Grotenhuis AJ, Dudek AM, Verhaegh GW, et al. Independent replication of published germline polymorphisms associated with urinary bladder cancer prognosis and treatment response. Bladder Cancer 2016;2:77-89.

10. Babjuk M, Böhle A, Burger M, et al. EAU guidelines on non-muscle-invasive urothelial carcinoma of the bladder: update 2016. Eur Urol 2017;71:447-61.

11. Lipunova N, Wesselius A, Cheng KK, et al. Genome-wide association study for tumour stage, grade, size, and age at diagnosis of non-muscle-invasive bladder cancer. Eur Urol Oncol 2019;2:381-9.

12. Zeegers MP, Bryan RT, Langford C, et al. The West Midlands Bladder Cancer Prognosis Programme: rationale and design. BJU Int 2010;105:784-8.

13. Hakonarson H, Gulcher JR, Stefansson K. deCODE genetics, Inc. Pharmacogenomics 2003;4:209-15.

14. Chang CC, Chow CC, Tellier LC, et al. Secondgeneration PLINK: rising to the challenge of larger and richer datasets. Gigascience 2015;4:7.

15. Purcell S, Neale B, Todd-Brown K, et al. PLINK: a tool set for whole-genome association and population-based linkage analyses. Am J Hum Genet 2007;81:559-75.

16. Loh PR, Danecek P, Palamara PF, et al. Reference-based phasing using the Haplotype Reference Consortium panel. Nat Genet 2016;48:1443-8.

17. Howie BN, Donnelly P, Marchini J. A flexible and accurate genotype imputation method for the next generation of genomewide association studies. PLoS Genet 2009;5:e1000529.

18. Clarke L, Fairley S, Zheng-Bradley X, et al. The international Genome sample resource (IGSR): A worldwide 
collection of genome variation incorporating the 1000 Genomes Project data. Nucleic Acids Res 2017;45:D854-9.

19. Johnson T, Kutalik Z. QUICKTEST user guide v0.94. 2008. Available online: http://toby.freeshell.org/software/ quicktest.shtml

20. Dayem Ullah AZ, Lemoine NR, Chelala C. SNPnexus: a web server for functional annotation of novel and publicly known genetic variants (2012 update). Nucleic Acids Res 2012;40:W65-70.

21. Dayem Ullah AZ, Lemoine NR, Chelala C. A practical guide for the functional annotation of genetic variations using SNPnexus. Brief Bioinform 2013;14:437-47.

22. Aken BL, Ayling S, Barrell D, et al. The Ensembl gene annotation system. Database (Oxford) 2016;2016:baw093.

23. GTEx Consortium. The Genotype-Tissue Expression (GTEx) project. Nat Genet 2013;45:580-5.

24. Schwender H, Selinski S, Blaszkewicz M, et al. Distinct SNP combinations confer susceptibility to urinary bladder cancer in smokers and non-smokers. PLoS One 2012;7:e51880.

25. Conti M, Richter W, Mehats C, et al. Cyclic AMP-specific PDE4 phosphodiesterases as critical components of cyclic AMP signaling. J Biol Chem 2003;278:5493-6.

26. Page CP, Spina D. Phosphodiesterase inhibitors in the treatment of inflammatory diseases. Handb Exp Pharmacol 2011;(204):391-414.

27. Abdel-Magid AF. Selective Inhibitors of Phosphodiesterase 4B (PDE-4B) May Provide a Better Treatment for CNS, Metabolic, Autoimmune, and Inflammatory Diseases. ACS Med Chem Lett 2017;8:1132-3.

28. Rahnama'i MS, Ückert S, Hohnen R, et al. The role of phosphodiesterases in bladder pathophysiology. Nat Rev Urol 2013;10:414-24.

29. Agis-Torres Á, Recio P, López-Oliva ME, et al. Phosphodiesterase type 4 inhibition enhances nitric oxideand hydrogen sulfide-mediated bladder neck inhibitory neurotransmission. Sci Rep 2018;8:4711.

30. Dong H, Claffey KP, Brocke S, et al. Inhibition of breast cancer cell migration by activation of cAMP signaling. Breast Cancer Res Treat 2015;152:17-28.

31. Powers GL, Hammer KD, Domenech M, et al. Phosphodiesterase 4D inhibitors limit prostate cancer growth potential. Mol Cancer Res 2015;13:149-60.

32. Domvri K, Zarogoulidis K, Zogas N, et al. Potential synergistic effect of phosphodiesterase inhibitors with chemotherapy in lung cancer. J Cancer 2017;8:3648-56.

33. Cooney JD, Aguiar RC. Phosphodiesterase 4 inhibitors have wide-ranging activity in B-cell malignancies. Blood 2016;128:2886-90.
34. Suhasini AN, Wang L, Holder KN, et al. A phosphodiesterase 4B-dependent interplay between tumor cells and the microenvironment regulates angiogenesis in B-cell lymphoma. Leukemia 2016;30:617-26.

35. Tsunoda T, Ota T, Fujimoto T, et al. Inhibition of phosphodiesterase-4 (PDE4) activity triggers luminal apoptosis and AKT dephosphorylation in a 3-D coloniccrypt model. Mol Cancer 2012;11:46.

36. Ou Y, Zheng X, Gao Y, et al. Activation of cyclic AMP/ PKA pathway inhibits bladder cancer cell invasion by targeting MAP4-dependent microtubule dynamics. Urol Oncol 2014;32:47.e21-8.

37. Smith PG, Wang F, Wilkinson KN, et al. The phosphodiesterase PDE4B limits cAMP-associated PI3K/AKT-dependent apoptosis in diffuse large B-cell lymphoma. Blood 2005;105:308-16.

38. Gong S, Chen Y, Meng F, et al. Roflumilast restores cAMP/ PKA/CREB signaling axis for FtMt-mediated tumor inhibition of ovarian cancer. Oncotarget 2017;8:112341-53.

39. Du B, Altorki NK, Kopelovich L, et al. Tobacco smoke stimulates the transcription of amphiregulin in human oral epithelial cells: evidence of a cyclic AMP-responsive element binding protein-dependent mechanism. Cancer Res 2005;65:5982-8.

40. Zuo WL, Yang J, Gomi K, et al. EGF-Amphiregulin Interplay in Airway Stem/Progenitor Cells Links the Pathogenesis of Smoking-Induced Lesions in the Human Airway Epithelium. Stem Cells 2017;35:824-37.

41. Bryan RT, Hussain SA, James ND, et al. Molecular pathways in bladder cancer: part 1. BJU Int 2005;95:485-90.

42. Burger M, Catto JW, Dalbagni G, et al. Epidemiology and risk factors of urothelial bladder cancer. Eur Urol 2013;63:234-41.

43. Park H, Yoon Y, Suk S, et al. Effects of different target sites on antisense RNA-mediated regulation of gene expression. BMB Rep 2014;47:619-24.

44. Furusawa T, Cherukuri S. Developmental function of HMGN proteins. Biochim Biophys Acta 2010;1799:69-73.

45. Murta-Nascimento C, Schmitz-Dräger BJ, Zeegers MP, et al. Epidemiology of urinary bladder cancer: from tumor development to patient's death. World J Urol 2007;25:285-95.

Cite this article as: Lipunova N, Wesselius A, Cheng KK, van Schooten FJ, Bryan RT, Cazier JB, Zeegers MP. Geneenvironment interaction with smoking for increased nonmuscle-invasive bladder cancer tumor size. Transl Androl Urol 2020;9(3):1329-1337. doi:10.21037/tau-19-523 


\section{Supplementary}

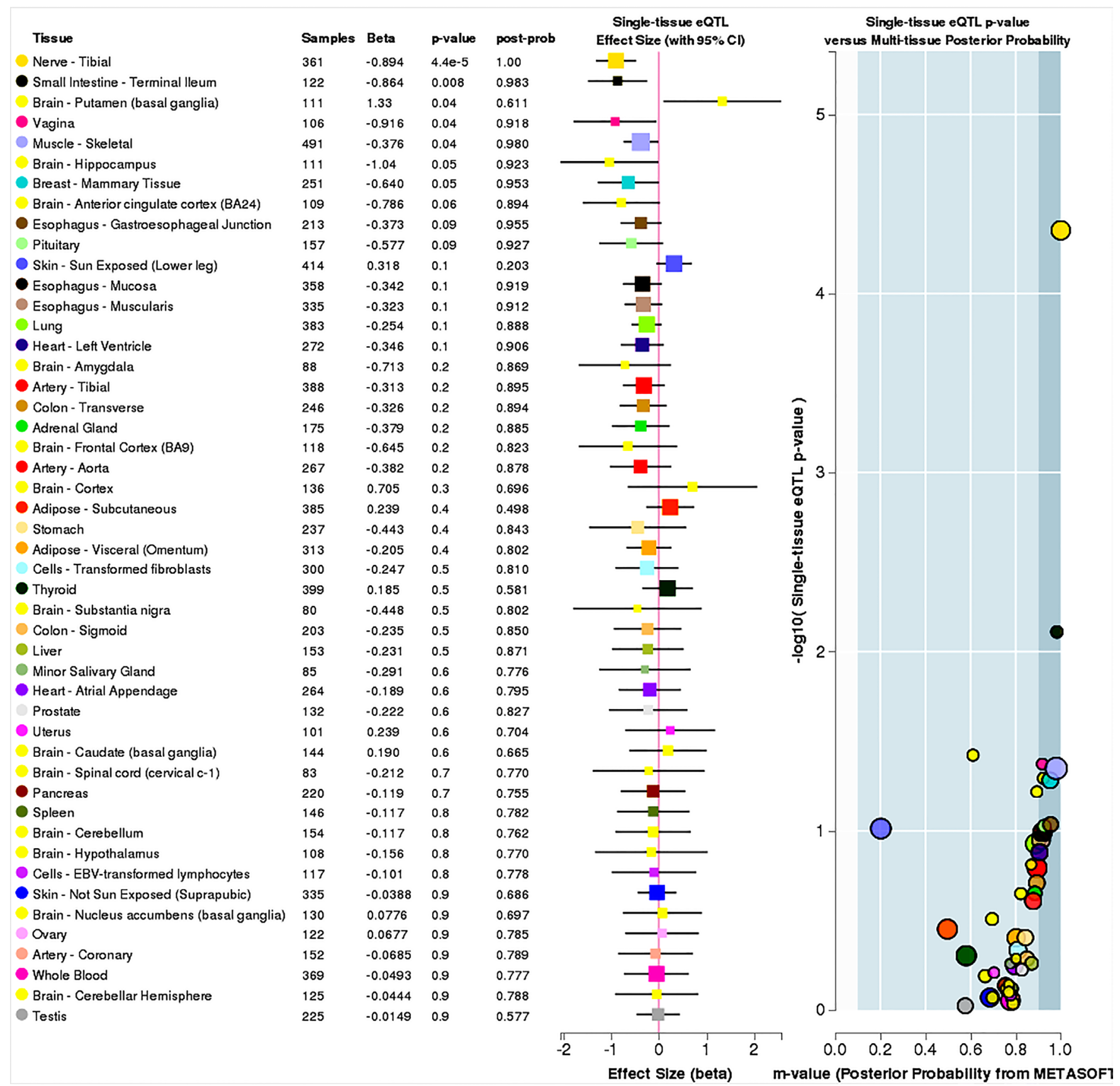

Figure S1 eQTL effect of rs144383242 (6q14.1) across multiple tissues (source: GTEx). eQTL, expression quantitative loci; GxE, geneenvironment interaction. 
Nerve_Tibial eQTL6_79489625_G_T_b37 ENSG00000270362.1

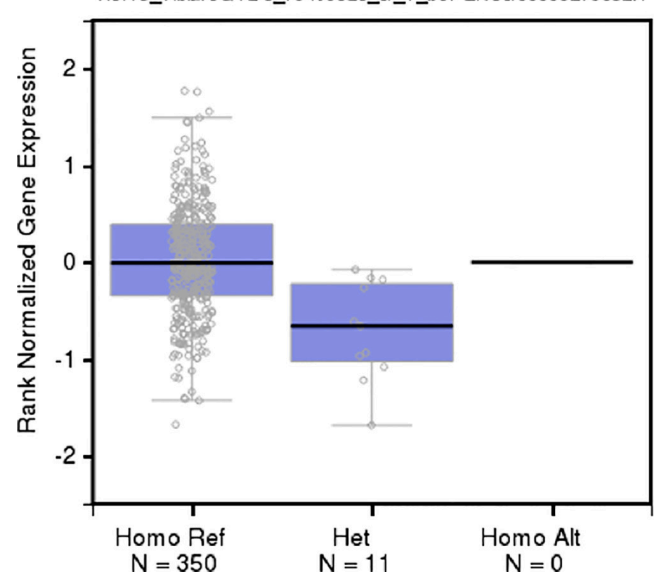

Figure S2 Boxplot of an eQTL effect of rs144383242 (6q14.1) in nerve tissue (source: GTEx). eQTL, expression quantitative loci; GxE, gene-environment interaction. 